\title{
Research on methods of technical support equipment maintainability verification based on Stratified random sampling
}

\author{
Dong Zhang ${ }^{1, a}$, Aihua $\mathrm{Xu}^{2}$,Yanmei Lv ${ }^{1}$ and Yanlong $\mathrm{Ni}$ \\ ${ }^{1}$ Mechanical Technology Institute, Shijiazhuang City, Hebei Province, China \\ ${ }^{2}$ Wuhan Mechanical Technology College, Wuhan City Hubei Province, China \\ a310792132@qq.com
}

Keywords: maintainability verification, stratified random sampling, technical support equipment.

\begin{abstract}
This paper introduced a type of maintainability verification method based on stratified random sampling, which improve the representativeness of the sample and the accuracy of final conclusions. Then deduced the method for determining samples quantity and test conclusions. Finally, take a certain type of technical support equipment maintainability verification as an example to verify the effectiveness of the method put forward in the paper.
\end{abstract}

\section{Introduction}

Maintainability is an important design feature of modern technical support equipment which affects the equipment support ability directly. Mean time to repair (MTTR) is a core indicator to measure the maintainability of equipments. Testing results of MTTR has become an important basis for evaluating maintainability. Maintainability verification means that times the maintenance and records the results in natural fault or simulated fault conditions and then computes the MTTR, and then verify whether the maintainability meets the requirements.

\section{Test sample selection method based on stratified random sampling}

Sample sources of maintainability verification are mainly two types: one is equipment faults and maintenance records in functional test, reliability test or environmental test. The other type is to simulate failures, which is to generate artificial faults when quantity of natural faults cannot meet the requirements of the test.

Commonly used sampling techniques in maintainability verification includes: simple random sampling, stratified random sampling, multi-stage sampling, cluster sampling, systematic sampling and non-probability sampling and so on. And characteristics of stratified random sampling are:

(1) Suitable for the equipments whose number of units is large.

(2) Suitable for the occasion when components of the equipments may exist significant difference in sampling problems.

(3) Stratified random sample can improve the accuracy of estimation of the indicators, because the population is generally divided into sub-layers which are very similar in composition. So small samples obtained within the sub-layer layer mean strong representation because of the similarity of each unit in the sub-layer.

(4) Sub-population parametric estimation can be done simultaneously.

The procedure of stratified random sampling is as follows:

(1) stratify the population into different layers according to the main technical characteristics of the components, the equipment can be divided into: sub-system mainly comprises electronic devices, sub-system mainly comprises the mechanical equipment, sub-system of the system mainly 
comprises optical devices and sub-system comprises integrated systems using a variety of techniques.

(2) Analyze maintenance operations of the equipments before the second stratification. Factors to be considered include: accessibility of maintenance object, environmental of maintenance operations (cabin operations or field operations), technical characteristics of maintenance (including main activities of maintenance, fault diagnosis techniques (BIT, ATE or artificial diagnostics, etc.), weight of the maintenance object, the number of fasteners and so on. Take electronic equipment as an example, organization level maintenance includes: fault diagnosis, removal, replacement and equipment debugging, among which fault diagnosis may be the biggest factor affecting the time of repairing, and then the technology used for fault diagnosis are main considerations for maintenance operations stratification. And meanwhile mechanical equipments usually use artificial diagnostic techniques, so time consumed on fault diagnosis has very little difference among people meeting the prescribed technical level and experience. Time of removal and replacement of fault components is a major determinant of the whole maintenance time. Factors like maintenance environment, maintenance equipment, maintenance objects accessibility, weight of maintenance object, the number of fasteners and so on should be major consideration in stratification.

(3) The third stratification usually takes frequency of maintenance, expected time-consuming and number of products as the main considerations, and the stratification process is the same as the corresponding provision in GJB.

\section{Determine the amount of test samples}

Because the probability distributions of the time cost in maintenance are different, the methods to determine the amount of test samples are different too. Based on past experience and statistical rules, maintenance time distribution of electronic technical support equipment generally conforms to normal distribution.

Set $\mathrm{X}$ the random variable of maintenance time, and $X \sim N\left(\mu, \sigma^{2}\right), \mu$ and $\sigma^{2}$ are mathematical expectation and variances. $X_{1}, X_{2} \ldots, X_{n}$ are samples of $\mathrm{X}$, then $\bar{X}=\frac{1}{n} \sum_{i=1}^{n} X_{i}$ is the maximum likelihood estimation of $\mu$, and $\bar{X} \sim N\left(\mu, \frac{\sigma^{2}}{n}\right)$. Here we discuss the problem in two cases, one is when $\sigma$ is known and the other is when $\mu$ is unknown.

(1) $\sigma$ is known

When $\sigma$ is known, $\frac{\bar{X}-\mu}{\sigma / \sqrt{n}} \sim N(0,1)$.

Set $P\{|\bar{X}-\mu| \leq \delta\}=1-\alpha$

Means $P\left\{\frac{|\bar{X}-\mu|}{\sigma / \sqrt{n}} \leq \frac{\delta}{\sigma / \sqrt{n}}\right\}=1-\alpha$, then we have: 
$\frac{\delta}{\sigma / \sqrt{n}}=\Phi(1-\alpha / 2)$

In equation (1) $\Phi(1-\alpha / 2)$ is the $1-\alpha / 2$ quantile of standard normal distribution. Then we have :

$n=\frac{\Phi^{2}(1-\alpha / 2) \sigma^{2}}{\delta^{2}}$

(2) When $\sigma$ is unknown.

When $\sigma$ is unknown, it is impossible to form a fixed length and a predetermined confidence level with a single sample quantity, criteria to determine the sample size must be different.

Set $P\{|\bar{X}-\mu| \leq k \sigma\}=1-\alpha$

In which: $\mathrm{k}$ is a given confidence interval, namely:

$P\left\{\frac{|\bar{X}-\mu|}{\sigma / \sqrt{n}} \leq k \sqrt{n}\right\}=1-\alpha$

Similar to the formula (3),

$$
n=\frac{\Phi^{2}(1-\alpha / 2)}{k^{2}}
$$

\section{Determine the test result}

After the test, in accordance with GJB2027 Maintainability Test and Evaluation, calculate the one-sided confidence limit of Mean Time To Repair. If the actual up-side confidence limit is less than or equal to the specified time requirements, maintainability quantitative indicators meet requirements. Otherwise, the MTTR does not meet the requirements.

The process is as follows.

Assuming the sample obtained $X_{1}, X_{2} \ldots, X_{n}$, the point estimate of repair time is:

$\bar{X}=\frac{1}{n} \sum_{i=1}^{n} X_{i}$

Point estimate of the variance is:

$\hat{d}^{2}=\frac{1}{n-1} \sum_{i=1}^{n}\left(X_{i}-\bar{X}\right)^{2}$

$X_{i}$ : the $\mathrm{i}$-th sample of random variable $\mathrm{X}$;

$n$ : test sample size;

$\bar{X}$ : mean value of samples;

$\hat{d}^{2}$ : sample variance.

Up-side confidence limit of mean time to repair: 
$\bar{X}_{u}=\bar{X}+Z_{1-\alpha} \frac{\hat{d}}{\sqrt{n}}$

$Z_{1-\alpha}: 1-\alpha$ quantile of normal distribution;

$\alpha:$ risk factor in the contract.

If $\bar{X}_{u} \leq$ MTTR , then the maintainability of the equipment meets the requirements. Otherwise, the maintainability of the equipment does not meet the requirements.

\section{Maintainability verification of some technical support equipment}

The technical support equipment mainly composed of five parts: chassis, shelter, power supply and distribution systems, electronic test platform and communication platform. In accordance with the requirements of stratified random sampling method to select the test samples, and common faults stratified results of the equipment are shown below.

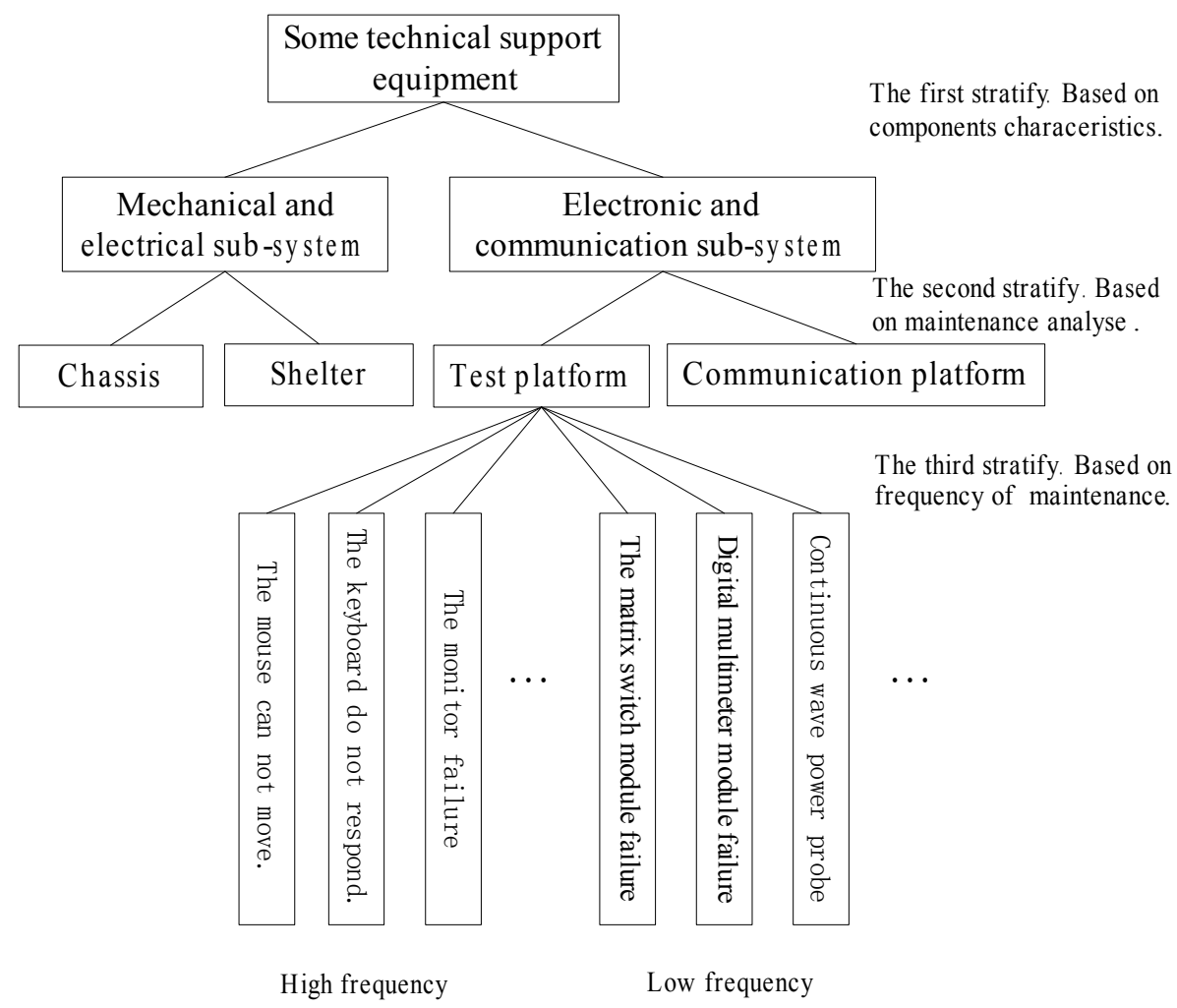

Figure.1 Stratify of the equipment

To classify the test sample set according to the method mentioned above, then calculate the sample size in accordance with contract requirements of risk level $\alpha$. Select from a variety of sample concentration corresponding to the number of samples for testing, record maintenance time, process test results in accordance with the formula described in the text, and then test conclusions can be drawn.

\section{Conclusions}

With the increasing complexity of technical level of equipment, the difficulty of maintenance 
support is increasing and equipment maintainability indicators are taken more and more seriously. The paper firstly gives a stratified random sampling method to selected test samples to improve the credibility of the samples and the accuracy of the test conclusions. Then the formula for determining the number of samples and test conclusions for technical support equipment are derived. Methods given in the paper provides basis for maintainability verification of technical support equipments.

\section{References}

[1] Study of the Method to Determine the Test Sample Size in Maintainability Test, Yu Lu, Tactical Missile Technology, May, 2008, (3): $29 \sim 32$.

[2] Sample Problem in Maintainability Demonstration Test, Wang Weilong, Automatic Measurement and Control, 2007, Vol.26, No.10.

[3] Study of Equipment MTTR on Random Weighted Method, Zhang Shouyu, Journal of Academy of Equipment Command and Technology, 2009, Vol.20, No.3.

[4] Research of Small Example Maintainability Experimentation and Evaluation of Bayes-Based Theory, Zhao Liang, Ship Electronic Engineering, Vol.26, No.1, $113 \sim 117$. 